\title{
COMPLETE IDEALS AND MONOIDAL TRANSFORMS
}

\author{
ARTHUR MATTUCK ${ }^{1}$
}

\begin{abstract}
It is proved that the monoidal transform of an integral noetherian scheme with respect to a sheaf $I$ of ideals is normal if and only if high powers of $I$ are complete. The analogous theorem for linear systems is included, and as an application, it is proved that a rational singularity is absolutely isolated.
\end{abstract}

A key geometric result of the elementary theory of linear systems is that a projective variety is normal if and only if the hypersurface sections of high degree form a complete linear system. This theorem has an exact analogue in the theory of complete ideals: the monoidal transform of a noetherian domain with respect to an ideal $I$ is normal if and only if high powers of $I$ are complete. Though this result is implicitly contained in the appendix to Zariski-Samuel which works out the two parallel theories, as well as in papers of Muhly, Nagata, and especially Rees [3], it does not seem to be explicitly stated and proved anywhere. We do so here, treating the two theories together; the proof seems new even in the linear systems case. The only technical tool needed is the notion of superficial element, though projective cohomology could be used instead.

As an application, we prove that the first neighborhood of an isolated rational singularity of a surface is always normal. ${ }^{2}$ This shows that such a singularity has only isolated singularities in its successive neighborhoods, never multiple curves; for a rational double point in characteristic zero, this was proved by Brieskorn.

1. Algebraic formulation. We recall briefly the essential parts of Zariski-Samuel [2]. Suppose one has a field $K$ containing both a

Received by the editors September 8, 1969.

AMS 1970 subject classifications. Primary 13B20, 14A05, 14A25, 14J15; Secondary $13 \mathrm{C} 99$.

Key words and phrases. Monoidal transform, quadratic transform, complete ideal, rational singularity, normal variety.

${ }^{1}$ Work supported by the National Science Foundation while the author was visiting at Brandeis University (1967/68), whose hospitality is gratefully acknowledged.

${ }^{2}$ ADDED IN PROOF. The long paper of Lipman, Rational singularities with applications to algebraic surfaces and unique factorization, [Inst. Hautes Etudes Sci. Publ. Math. No. 36 (1969) ] has just appeared. In it this theorem (among many others) is stated and proved. The first two sections of this paper heavily intersect Lipman's; he uses a different method to establish the completeness of $m^{n}$ for a rational singularity. 
domain $A$ and an $A$-module $I$. The integral closure of $I$ in $K$ is

$$
I^{\prime} \equiv\left\{z \in K \mid z^{n}+a_{1} z^{n-1}+\cdots+a_{n}=0, a_{i} \in I^{i}\right\}
$$

and by an elementary theorem, this is also the completion of $I$ in $K$ :

$$
I^{\prime} \equiv \bigcap_{v \in \delta} R_{v} I, \quad \delta=\left\{v \mid R_{v} \supseteq A\right\},
$$

where $R_{v}$ is the valuation ring of the valuation $v$ of $K$.

We in troduce the graded algebras (we take $I^{0}=A$ )

$$
R=\coprod_{n \geq 0} I^{n}, \quad S=\coprod_{n \geq 0}\left(I^{n}\right)^{\prime} .
$$

Note that (2) shows that $\left(I^{m}\right)^{\prime}\left(I^{n}\right)^{\prime} \subseteq\left(I^{m+n}\right)^{\prime}$, so that $S$ is a graded $R$ algebra.

Let $x_{1}, x_{2}, \ldots$ be a set of nonzero generators for $I$, and consider the localizations

$$
R_{i}=\left\{\frac{F}{x_{i}^{k}} \mid F \in I^{k}\right\}, \quad S_{i}=\left\{\frac{G}{x_{i}^{k}} \mid G \in\left(I^{k}\right)^{\prime}\right\} .
$$

Then $R_{i}$ and $S_{i}$ are subrings of $K$, and a routine argument using the integral equations (1) shows that

$$
S_{i}=R_{i}^{\prime} .
$$

Since $x_{j_{1}} \cdots x_{j_{n}}=\left(x_{j_{1}} / x_{i}\right) \cdots\left(x_{j_{n}} / x_{i}\right) x_{i}^{n}$, one has in $K$

$$
R_{i} I^{n}=R_{i} x_{i}^{n}, \quad S_{i} I^{n}=S_{i} x_{i}^{n}, \quad n \geqq 0 .
$$

Proposition 1. If I is of finite type, $\left(I^{n}\right)^{\prime}=\bigcap_{i} S_{i} I^{n}, n \geqq 0$.

Proof. This is essentially [2, p. 354, Lemma]. Let $S_{i}$ be the set of valuations of $K$ such that $R_{v} \supseteq R_{i}$. Then we claim $\delta=U S_{i}$, for if we are given any $v \in \mathcal{S}$, we find that index $i$ for which $v\left(x_{i}\right)$ is minimum. Then $v\left(x_{v} / x_{i}\right) \geqq 0$ for all $\nu$, so $v \in S_{i}$. Thus

$$
\left(I^{n}\right)^{\prime}=\bigcap_{i} \bigcap_{v \in s_{i}} R_{v} I^{n}=\bigcap_{i} \bigcap_{v \in S_{i}} R_{v} x_{i}^{n}=\bigcap_{i} S_{i} x_{i}{ }^{n}
$$

according to (2), (6), and (5).

Proposition 2. If $I$ is of finite type and $A$ is noetherian,

$$
I^{n}=\bigcap_{i} R_{i} I^{n} \text { for } n \geqq n_{0} .
$$

Proof. This is really projective cohomology, but one can use superficial elements instead. Recall [3] that if $\mathfrak{a}$ is an ideal in a noetherian 
domain, $z \in \mathfrak{a}^{d}$ is superficial of degree $d$ for $\mathfrak{a}$ if

$$
\mathfrak{a}^{n+d}: z=\mathfrak{a}^{n} \text { for } n \geqq n_{0} .
$$

If $z$ is such an element, then it follows immediately that

$$
\mathfrak{a}^{n+d k}: z^{k}=\mathfrak{a}^{n} \quad \text { for } n \geqq n_{0}, k>0 .
$$

Superficial elements exist, though not necessarily for $d=1$.

The proof of the proposition takes place inside $R$. Let $I$ be generated by $x_{1}, \cdots, x_{m}$. Then in $R$ we have the ideals

$$
\bar{I}=\coprod_{n>0} I^{n}, \quad \bar{I}^{k}=\coprod_{n \geq k} I^{n}
$$

$R$ is noetherian, since it is finitely generated (by the $x_{i}$ ) over $A$, and it is also a graded subring of the graded ring $\bar{K}=\coprod \bar{K}_{(n)}$, where $\bar{K}_{(n)}=K$ for all $n \geqq 0$. To prove the nontrivial inclusion $\supseteq$ of the proposition, let $z \in \bar{I}^{d}$ be superficial of degree $d$ for $\bar{I}$, and $n_{0}$ be as in (7). Suppose given $y \in K$ such that

$$
y \in R_{i} I^{n}, \quad n \geqq n_{0}, \text { all } i .
$$

We view $y$ as an element of $\bar{K}_{(n)}$ in what follows. For some common exponent which we may take to be a multiple $k d$ of the integer $d$,

$$
x_{i}^{k d} y \in \bar{I}^{n+k d}, \quad n \geqq n_{0} \text {, all } i .
$$

Since $z \in \bar{I}^{d}$, it follows in the usual way that if $m$ is large

$$
z^{k m} y \in \bar{I}^{n+k d m}, \quad n \geqq n_{0} .
$$

Therefore $y \in \bar{I}^{n}$, according to (8). Thus $y \in \bar{I}^{n} \cap \bar{K}_{(n)}=I^{n}$. (If one is only interested in the case $I \subseteq A$, the same proof can be carried out entirely inside $A$.)

2. Geometric formulation. We translate the preceding section into geometry, using the simplest properties of the functor Proj on commutative graded algebras [4]. Let $Y$ denote $\operatorname{Spec} A$, and let $V$ be Proj $R$ and $W$ denote Proj $S$, so that we have the dual diagrams

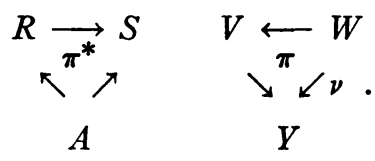

Since $R$ is generated over $A$ by the $x_{i}$, the definition of Proj $R$ shows that $V$ is covered by the affines $V_{i}=\operatorname{Spec} R_{i}$. It is easily checked that 
$\pi^{-1}\left(V_{i}\right)=W_{i}=\operatorname{Spec} S_{i}$. Then (5) shows that $W_{i}$ is the normalization of $V_{i}$, so that without finiteness assumptions,

$$
\text { Proj } S \text { is the normalization in } K \text { of Proj } R \text {. }
$$

THEOREM 1. If $I^{n}$ is complete in $K$ for large $n$, then Proj $R$ is normal. The converse holds, provided $A$ is noetherian, $I$ is of finite type, and $K$ is the quotient field of $A\left[\cdots, x_{i} / x_{j}, \cdots\right]$.

Proof. If $I^{n}=\left(I^{n}\right)^{\prime}$, then $\pi^{*}$ is an isomorphism in high degree. An elementary property of Proj shows that $\pi$ is then an isomorphism, so $V$ is normal, by (10). Conversely, if $V$ is normal and $K$ is its function field, then by (10), $V=W$. Thus $V_{i}=W_{i}$ and $R_{i}=S_{i}$ for all $i$, so that $I^{n}=\left(I^{n}\right)^{\prime}$ by Propositions 1 and 2 , if $n \geqq n_{0}$.

Let $Y$ be an integral noetherian scheme, $g$ a coherent sheaf of fractional ideals on $Y$. The $Y$-scheme $V=\operatorname{Proj}\left(\amalg^{n}\right)$ is then called the monoidal transform of $Y$ with respect to $g[4,8.1 .3]$. To apply Theorem 1, we take $K$ to be the function field of $Y$.

The completion $g^{\prime}$ we define to be the sheaf such that $\Gamma\left(U, g^{\prime}\right)$ $=\Gamma(U, g)^{\prime}$ for any affine $U \subset Y$. This defines a sheaf, as one sees easily using (2). If we now set $W=\operatorname{Proj}\left(\Pi\left(g^{n}\right)^{\prime}\right)$, then $W$ is the normalization of $V$. Routine globalization plus Proposition 1 gives

THEOREM 2. The monoidal transform $V$ of $Y$ with respect to $g$ is a normal scheme if and only if $\mathrm{g}^{n}$ is complete for $n$ large. If $W$ is the normalization of $V$, and $\nu: W \rightarrow Y$ the structural map,

$$
\nu * \nu^{*} g^{n}=\left(g^{n}\right)^{\prime} \text { for } n \geqq 0 .
$$

Consider the case of a linear system on a $k$-variety $X$, now. In Theorem 1, we take $Y=\operatorname{Spec} k$ and $K=k(X)$. The module $I$ is then a finite-dimensional $k$-space of functions on $X$, generated say by $x_{0}, \cdots, x_{n}$. Then $R=k\left[t x_{0}, \cdots, t x_{n}\right]$ where $t$ is a transcendental, $V$ is a projective $k$-variety, and since the $x_{i}$ are functions on $X$, we get a dominating rational map $\phi: X \rightarrow V$.

Theorem 1 then says that $V$ is normal if $I^{n}$ is complete for large $n$, with the converse holding if for instance $\phi$ is birational. As a special case, we could take $\phi$ to be the identity map; this will be so when $X=\operatorname{Proj} k\left[t_{0}, \cdots, t_{n}\right]$ and we take $I$ to be the subspace generated by the functions $t_{i} / t_{0}$ (assuming $t_{0}$ transcendental over the $t_{i}$ ). In view of the usual definitions, we conclude that $X$ is normal if and only if the linear system of hypersurface sections of degree $n$ is complete for large $n$.

As another example, assume $X$ is normal, $D$ a divisor on it, and take $I=\{f \in K \mid f \geqq-D\}$. Then $I$ is complete [2, p. 358], one calls $\phi$ customarily the "rational map defined by $|D|$, , and we conclude 
that the image of a normal variety under the rational map determined by a complete linear system is normal.

3. Application to rational singularities. We take a two-dimensional normal local domain $A$ with maximal ideal $\mathfrak{m}$ and algebraically closed residue field. Put $Y=\operatorname{Spec} A$ and let $p$ be the closed point; it is an isolated singularity of $Y$ since $A$ is normal. Such a singularity can be resolved by a sequence of monoidal transformations, each followed by a normalization, if needed. This process leads ultimately to a nonsingular scheme $\bar{W}$ with a proper birational morphism

$$
h: \vec{W} \underset{\mu}{\rightarrow} W \underset{\nu}{\rightarrow} Y
$$

which factors through $W$, the normalized monoidal transform of $Y$ with respect to $m$. Theorem 2 may now be extended to show that

$$
h_{*} h^{*} \mathrm{~m}^{n}=\left(\mathrm{m}^{n}\right)^{\prime} \text { for all } n \geqq 0 .
$$

To see this, remark first that since $W$ is the normalized monoidal transform, it follows from (6) that $g_{n}=\nu^{*} \mathfrak{m}^{n}$ is an invertible sheaf of ideals. Now we claim that $\mu_{*} \mu^{*} g_{n}=g_{n}$ for all $n \geqq 0$. For since this assertion is local on $W$, we may assume $\partial_{n}=\theta_{W}$, in which case it says, if $f$ is in the quotient field of $A$, then

$$
\mu^{*} f \in \Gamma\left(\bar{W}, \boldsymbol{\theta}_{\bar{W}}\right) \Rightarrow f \in \Gamma\left(W, \boldsymbol{\theta}_{W}\right) .
$$

This is true, for if $f$ were not holomorphic on $W$, it would have a polar divisor (since $W$ is normal), hence so would $\mu^{*} f$.

Putting this together with Theorem 2 proves (11), since

$$
h_{*} h^{*} \mathfrak{m}^{n}=\nu_{*} \nu^{*} \mathfrak{m}^{n}=\left(\mathfrak{m}^{n}\right)^{\prime} .
$$

Suppose now that $p$ is a rational singularity. By definition, this means that $R^{1} h_{*} \mathcal{O}_{\bar{W}}=0$, i.e., that the singularity contributes nothing to the arithmetic genus of the surface. In [1], Artin studies the divisor $Z=h^{-1}(p)$, and one of his key results is

For all $n>0, \quad$ if $f \in A$ and $h^{*} f \geqq n Z, \quad$ then $f \in \mathfrak{m}^{n}$.

In other words: for all $n>0, h_{*} h^{*} \mathfrak{m}^{n}=\mathfrak{m}^{n}$. Comparing with (11) shows that for a rational singularity, $\mathrm{m}^{n}$ is complete for $n>0$. Therefore by Theorem 2, its monoidal transform is normal and has only isolated singularities. Since the Leray sequence for $\bar{W} \rightarrow W \rightarrow Y$ shows that these too are also rational, it follows by induction that the rational singularity is absolutely isolated, i.e., the successive steps in the resolution process produce only isolated singularities, never multiple curves. 


\section{REFERENCES}

1. M. Artin, On isolated rational singularities of surfaces, Amer. J. Math. 88 (1966), 129-136. MR 33 \#7340.

2. O. Zariski and P. Samuel, Commutative algebra. Vol. 2, University Series in Higher Math., Van Nostrand, Princeton, N. J., 1960. MR 22 \#11006.

3. D. Rees, Valuations associated with ideals, Proc. London Math. Soc. (3) 6 (1956), 161-174. MR 17, 1047.

4. A. Grothendieck, Elements de géométrie algêbrique, Inst. Hautes Etudes Sci. Publ. Math. No. 11 (1961). MR 36 \#177.

5. S. Lang, Introduction to algebraic geometry, Interscience, New York, 1958, p. 164. MR $20 \# 7021$.

6. E. Brieskorn, Rationale Singularitäten komplexer Flächen, Invent. Math. 4 (1967/68), 336-358. MR $36 \# 5136$.

Massachusetts Institute of Technology, Cambridge, Massachusetts 02139 